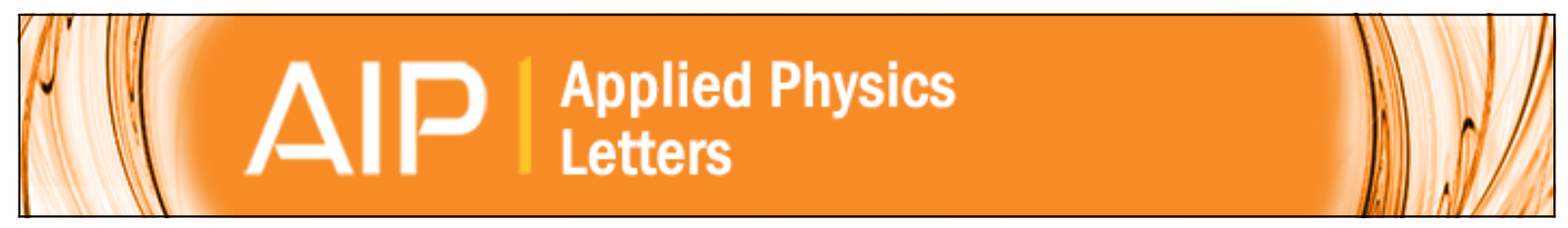

\title{
Polymer nanofibers by soft lithography
}

Dario Pisignano, Giuseppe Maruccio, Elisa Mele, Luana Persano, Francesca Di Benedetto, and Roberto

Cingolani

Citation: Applied Physics Letters 87, 123109 (2005); doi: 10.1063/1.2046731

View online: http://dx.doi.org/10.1063/1.2046731

View Table of Contents: http://scitation.aip.org/content/aip/journal/apl/87/12?ver=pdfcov

Published by the AIP Publishing

\section{Articles you may be interested in}

Fabrication of wafer-scale nanopatterned sapphire substrate by hybrid nanoimprint lithography

J. Vac. Sci. Technol. B 32, 06FG06 (2014); 10.1116/1.4898778

Recessed area patterning via nanoimprint lithography

J. Vac. Sci. Technol. B 29, 060602 (2011); 10.1116/1.3660393

Atomic step patterning in nanoimprint lithography: Molecular dynamics study

J. Vac. Sci. Technol. B 29, 06FC11 (2011); 10.1116/1.3659712

Soft photocurable nanoimprint lithography for compound semiconductor nanostructures

J. Vac. Sci. Technol. B 26, 156 (2008); 10.1116/1.2823035

Erratum: "Atomic force microscope based nanofabrication of master pattern molds for use in soft lithography" [Appl. Phys. Lett.91, 123111 (2007)]

Appl. Phys. Lett. 91, 239901 (2007); 10.1063/1.2813011

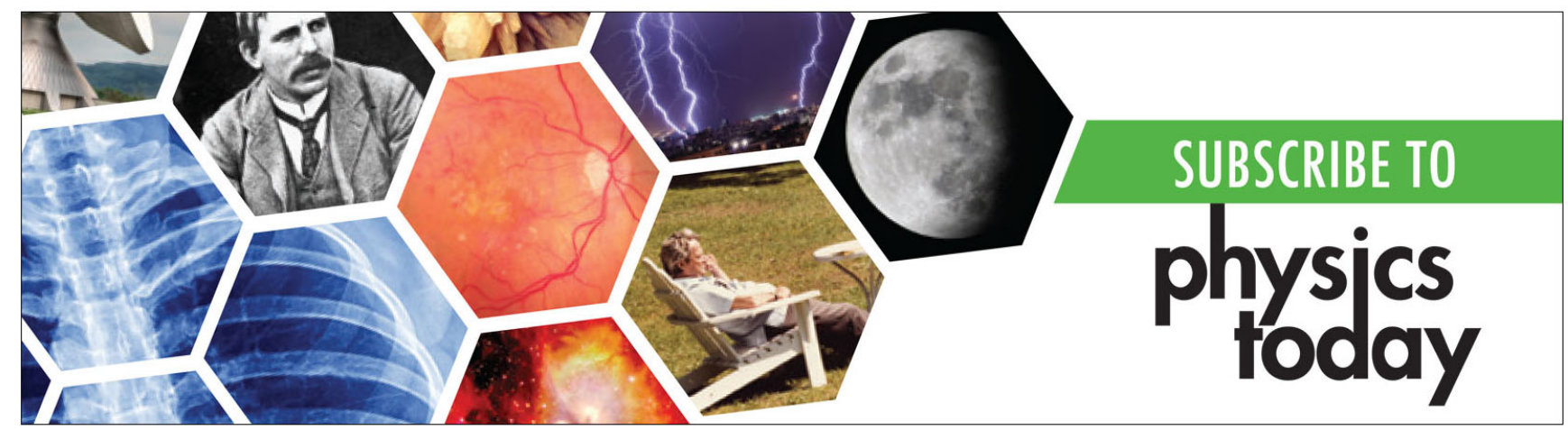




\title{
Polymer nanofibers by soft lithography
}

\author{
Dario Pisignano, ${ }^{\text {a) }}$ Giuseppe Maruccio, Elisa Mele, Luana Persano, \\ Francesca Di Benedetto, and Roberto Cingolani \\ NNL, National Nanotechnology Laboratory of INFM-CNR and Agilent Technologies Joint Lab clo \\ Dipartimento di Ingegneria dell'Innovazione, Università di Lecce, via Arnesano, I-73100 Lecce, Italy
}

(Received 19 April 2005; accepted 26 July 2005; published online 13 September 2005)

The fabrication of polymeric fibers by soft lithography is demonstrated. Polyurethane, patterned by capillarity-induced molding with high-resolution elastomeric templates, forms mm-long fibers with a diameter below $0.3 \mu \mathrm{m}$. The Young's modulus of the fabricated structures, evaluated by force-distance scanning probe spectroscopy, has a value of $0.8 \mathrm{MPa}$. This is an excellent example of nanostructures feasible by the combination of soft nanopatterning and high-resolution fabrication approaches for master templates, and particularly electron-beam lithography. (c) 2005 American Institute of Physics. [DOI: 10.1063/1.2046731]

Polymer micro- and nanofibers are raising increasing interest due to their smart properties for a wide class of applications, ranging from textiles and microelectromechanical systems to sensors ${ }^{1}$ and optoelectronics. ${ }^{2}$ Nanofibers are excellent building blocks for structurally reinforced composites, ${ }^{3}$ membranes with high filtration efficiency, ${ }^{4}$ and scaffolds for tissue engineering. They can be employed as porous elements for smart clothes and for protective wound dressing, by virtue of their ability to capture aerosol particles, ${ }^{5}$ and as large-surface supports for enzymes and catalysts. ${ }^{6}$ Polymeric nanofibers also find use as matrixes of electrolytes for high-performance batteries, ${ }^{7}$ sacrificial templates for nanofluidic channels, ${ }^{8}$ and active media for electronic and photonic devices, such as field effect transistors and optical polarizers. ${ }^{2}$

So far, the practically unique technique, which is able to fabricate polymeric nanofibers with relatively low cost and high throughput is electrospinning, which is based on the uniaxial elongation of a fluid polymer jet under an intense applied electric field. ${ }^{5}$ In this work, we demonstrate that high-resolution soft lithography is a parallel, cheap and straightforward approach for realizing polymeric fibers. Capillary-assisted molding, by composite elastomeric elements with features of suitable aspect-ratio $(>1)$, is demonstrated to produce self-detachable polyurethane fibers, with diameter of $265 \mathrm{~nm}$ and length of several mm, a size distribution of width as low as $15 \mathrm{~nm}$, and a Young's modulus of $0.8 \mathrm{MPa}$.

The fabrication process is schematized in Fig. 1. Dense patterns of periodic grooves were fabricated by electron beam lithography (EBL) by a Raith Elphy Plus pattern generator controlling a Leica Stereoscan 440 scanning electron microscope (SEM), and subsequent reactive ion etching by means of a $\mathrm{CF}_{4} / \mathrm{Ar}$ mixture. The master morphology is replicated by composite stamps of hard-PDMS ( $h$-PDMS) and PDMS,${ }^{9}$ i.e., a stiffer polymer layer supporting the pattern, covered by a softer layer favoring the conformal contact over a wider area (more than a few $\mu \mathrm{m}^{2}$ ) on the target surface. After placing the elastomeric elements on a previously spincast film of the target polymer (step a), the molding process is carried out (step b), through the simultaneous penetration and curing of the polymer by UV-irradiation. The separation

a) Author to whom correspondence should be addressed; electronic mail: Dario.pisignano@unile.it of the replica leads to the rearrangement of the shape of the realized aligned features, which finally detach from the substrate thus forming the fibers (steps c-e).

During molding, the elastomeric elements have to assure the conformal contact with the target substrate, thus enabling the soft lithography to be performed. ${ }^{10-12}$ To this aim, and to also accomplish high resolution, one has to find a trade off between the stiffness of the elastomeric structure (i.e., a high value of the Young's modulus, E) and the flexibility and conformability of the replicas. The former enhances the mechanical stability of the replicated features, thus allowing to reach higher aspect-ratios and resolutions. Molds too flexible (i.e., with low values of $E$ ) can suffer ample distortions (i.e., deformation and pairing of close features) and collapse of high-aspect ratio features by mutual adhesion. ${ }^{13}$ The flexibility of the replicas favors instead their intimate contact with the substrates. These parameters, which have to be optimized
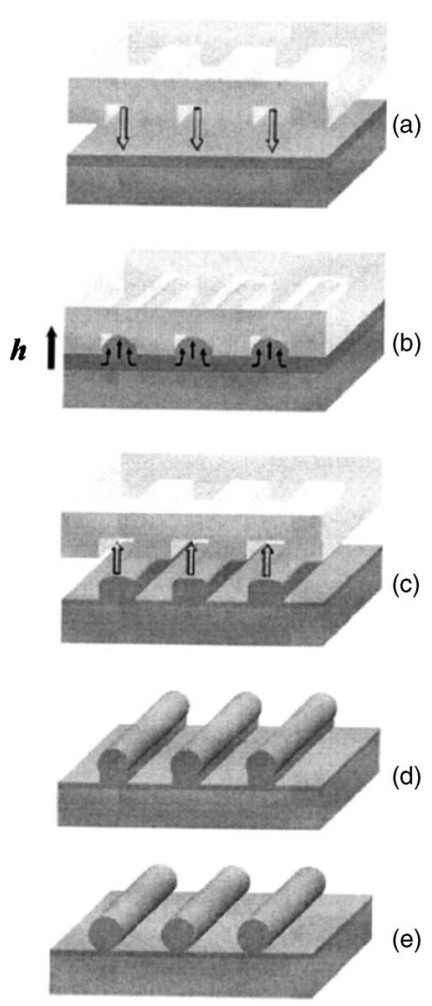

FIG. 1. Process flow for fabrication of polymer fibers by soft lithography. 

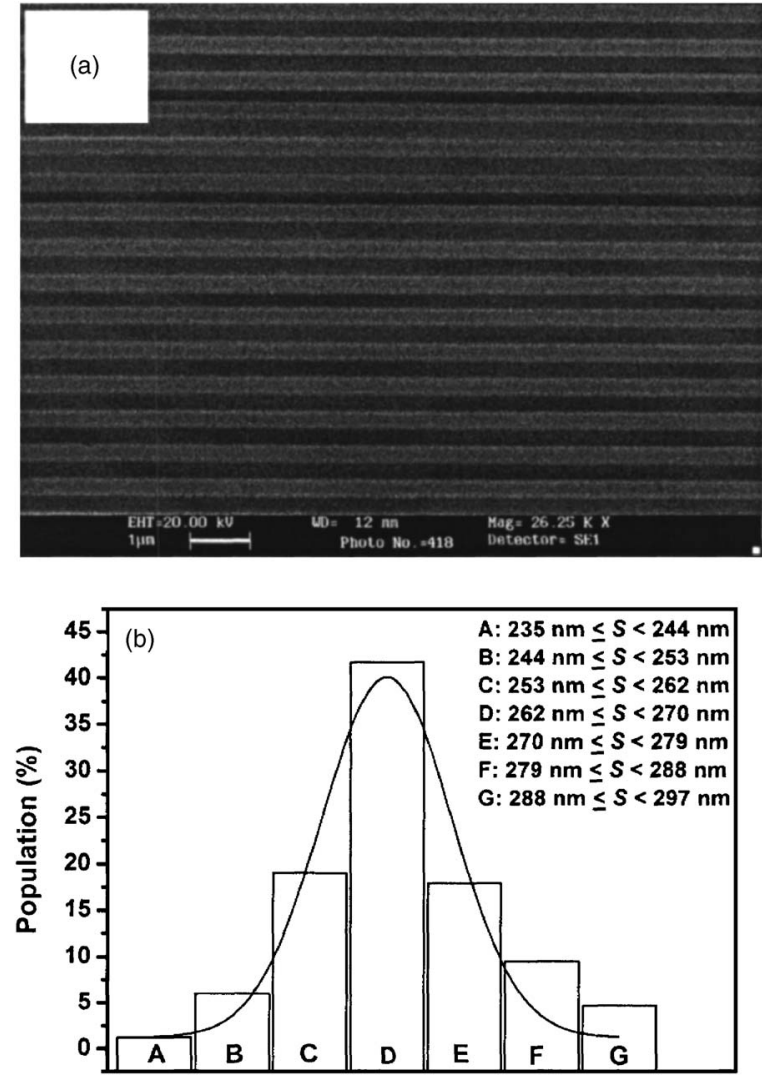

FIG. 2. (a) Aligned fiber pattern fabricated by capillary-assisted molding (b) Obtained size (i.e., section, $S$ ) distribution and its fit by a Gaussian curve (solid line).

depending on the used elastomer, led to the use of composite stamps.

The molding process can be performed on a wide range of polymers, including conjugated materials, resists, and epoxy-systems, and it can be assisted by heating, thus exploiting the glass-transition phenomenology of amorphous solids, or simply driven by vertical capillarity, like in the case of polymerisable materials. In this work, we focused on capillary-induced molding (i.e., vertical microfluidic lithography) of polyurethane, for which the pattern transfer from the PDMS element to the curable polymer is mainly due to the fluid penetration into the recessed features of the replica. Such a process is strongly enhanced by the low viscosity of the employed polymer, since the fluidic filling rate of the vertical channels depends on the viscosity of the polymer, $\eta$, according to the equation ${ }^{14}$

$$
\frac{d h}{d t}=\frac{R \gamma \cos \Theta}{4 \eta h},
$$

where $h$ is the vertical coordinate indicating the penetration into the recessed feature (Fig. 1), $R$ is the hydraulic radius of the capillary, $\gamma$ is the surface tension at the polymer/air interface (around $40 \mathrm{mN} / \mathrm{m}$ ), and $\Theta$ is the contact angle between the polymer and the surface of the capillary (around $80^{\circ}$ ). Equation (1) allows us to estimate the maximum height, $h_{m}$, that the polymer can reach within the time necessary for a complete polymerization. Since for $100-\mathrm{nm}$ scale features, the calculated value of $h_{m}$ is of the order of millimeters, this approach is a promising, flexible candidate for manufacturing nanostructures of high aspect-ratio. In particular, a high aspect-ratio is crucial to favour the cohesion forces of the polymer features with respect to the adhesion to
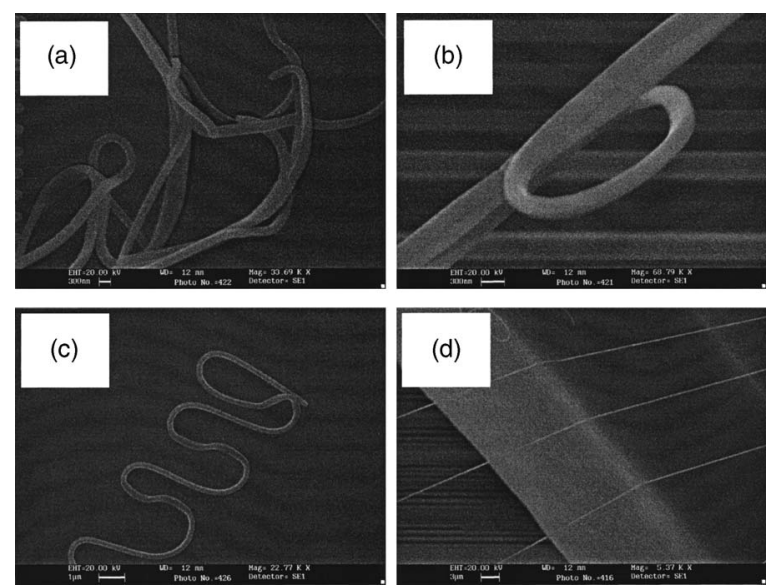

FIG. 3. Polymer fibers made by soft lithography. (a) Disordered bundle of fibers, very similar to those usually achieved by electrospinning. (b) Knot, evidencing the flexibility of the realized fibers and their rounded shape. (c) Single fiber, with diameter of about $300 \mathrm{~nm}$. (d) Nano-Gulliver. A $h$-PDMS fragment is anchored to the substrates by polyurethane fibers.

the substrate, thus promoting the detachment of the stripes from the solid surface (steps c-e in Fig. 1). Photocurable polyurethanes are particularly suitable for this process, since they can undergo weak adhesion to the substrates, as demonstrated by the free-standing patterned structures obtained by horizontal micromolding in capillaries. ${ }^{15}$

A resultant pattern of aligned polymer fibers is shown in Fig. 2. The very good pattern definition indicates that the achieved resolution depends on the starting masters: to date, features with lateral size down to $10 \mathrm{~nm}$ have been demonstrated by high-resolution mechanical lithography. ${ }^{16}$ Such a soft lithography approach allows a high control on:

position, by the direct fabrication of aligned fibers (Fig. 2), which is a major advantage over other existing methods, requiring complex subsequent aligning procedures;

(ii) size, depending on the master features. Our fibers have a size distribution centered at $266 \mathrm{~nm}$, with a width of $15 \mathrm{~nm}$ [full width half maximum, see Fig. 2(b)].

The throughput of the technique is determined mainly by the pattern area and density of the fabricated master. Like electrospinning, molding lithography is an inherently topdown nanofabrication approach. The parallel formation of many fibers is here directly primed by the templated capillary penetration. For instance, more than $1.5 \times 10^{4}$ fibers of size around $300 \mathrm{~nm}$ can be produced in a single molding by a $1 \times 1 \mathrm{~cm}^{2}$ stamp.

After detachment from the substrate, the fibers can be easily isolated and observed in a variety of configurations, suggesting a high degree of structural flexibility (Fig. 3). In order to investigate in depth the mechanical properties of our fibers, we performed force-distance spectroscopy by a scanning probe tip $(\mathrm{Si})$ on stripes in the aligned pattern of Fig. 2, thus determining the contact stiffness, $\kappa$, along the direction (z) perpendicular to the fiber surface: $\kappa=d F / d z$, where $F$ stands for the load applied to achieve an elastic deformation. A typical topographical atomic force micrograph of the fibers, and the corresponding loading-unloading force-distance cycle are shown in Figs. 4(a) and 4(b), respectively; a slight hysteresis is observed in the curves due to the adhesion be- 

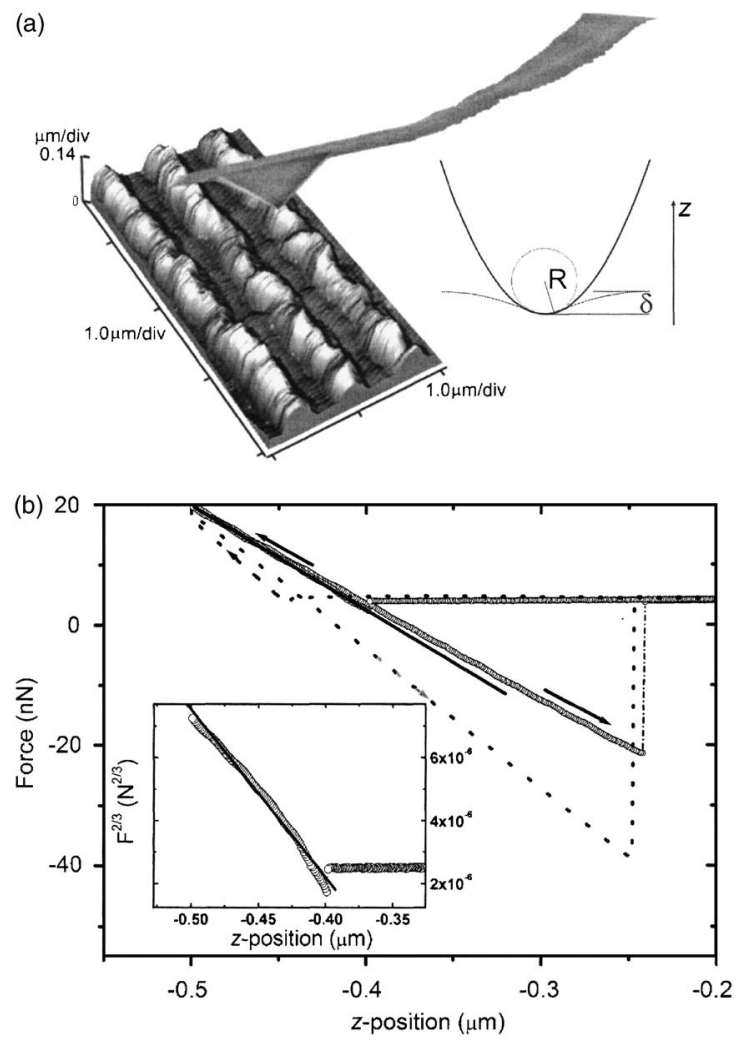

FIG. 4. (a) AFM image of fibers lying on silicon (like in step c of Fig. 1), and scheme of the probe tip (Silicon Veeco Ultralevers tips mounted on a Thermomicroscope-CP Research atomic force microscope) indicating the loading point on the top of the fibers. Inset: scheme of the elastic compression of the polymer surface, and of the $z$ coordinate system. $R$ : radius of the contact paraboloid, $\delta$ : indentation (b) Force-displacement curve on a polyurethane fiber (open circles), and on the bare silicon substrate (dotted curves). The arrows indicate the displacement direction for the different curves. Solid line: linear fit of the approaching curve on the polymeric fibers. The slope of the approach curve on $\mathrm{Si}$ instead is defined by the value of the spring constant of the employed tip $\left(0.26 \mathrm{~N} / \mathrm{m}, E_{t}=152 \mathrm{GPa}\right)$. Inset: $F^{2 / 3}$ vs vertical coordinate, $z$ (open circles), for the approach curve, and fitting line by Eq. (2). The proportionality constant, $\left(4 R^{1 / 2} E^{*} / 3\right)^{2 / 3}$, has a value of $5.3 \times 10^{-5} \mathrm{~N}^{2 / 3} / \mathrm{m}$.

tween the polymer and the withdrawing tip. Upon unloading, we could not observe any permanent deformation at the indentation points, which suggests that the applied stress $(<20 \mathrm{nN})$ is well within the elastic regime of the polymeric nanostructures. From the measured loading force-distance curve, we extracted a value of $\kappa$ of about $0.17 \mathrm{~N} / \mathrm{m}$ [fitting line in Fig. 4(b)]. We estimate the Young's modulus of the fibers using the following expression for an elastic sphereplane contact described by a Hertzian model [inset of Fig. $4(\mathrm{a})],{ }^{17,18}$

$$
F^{2 / 3}=\left(\frac{4 R^{1 / 2} E^{*}}{3}\right)^{2 / 3} \Delta z
$$

where $R$ is the relative radius of curvature of the tip-sample indentation, and $E^{*}$ is the reduced Young's modulus of the system,

$$
E^{*}=\left[\frac{\left(1-\nu_{t}\right)^{2}}{E_{t}}+\frac{\left(1-\nu_{s}\right)^{2}}{E_{s}}\right]^{-1}
$$

In the previous expression, $\nu_{t}$ and $\nu_{s}\left(E_{t}\right.$ and $\left.E_{s}\right)$ indicate the Poisson's ratio (Young's modulus) of the probe tip $(t)$ and of the sample $(s)$, respectively. Assuming that the tip does not undergo deformation upon contact (see the caption of Fig. 4), and taking into account the Poisson's ratio of the bulk polymer $\left(\nu_{s}=0.47\right)$, we find for the Young's modulus of the fibers a value of $0.8 \mathrm{MPa}$. For sake of comparison, we recall that this is more than twenty times smaller than the modulus for the bulk polymer (16.9 MPa). This is consistent with the large flexibility observed in our fibers (Fig. 3).

In conclusion, we have presented a method to realize polymeric nanofibers in a parallel way. The resulting fibers are initially aligned, and then can be easily separated from the starting substrate. Today, a wide number of novel nanofiber structures and compounds are being proposed, such as functional polymers, ${ }^{19}$ core-sheath, ${ }^{20}$ and hollow systems. ${ }^{21}$ Multilayer patterning methods, organic modifiers to tune the wetting properties of the involved surfaces, and unmixable polymeric fluids, can be certainly effectively employed to fabricate more complex fiber structures by soft lithography. The polymeric nanofibers are an excellent example of the novel perspectives open for soft nanopatterning by the combination with high-resolution fabrication approaches for master templates, and particularly with electron-beam lithography.

${ }^{1}$ X. Wang, C. Drew, S.-H. Lee, K. J. Senecal, J. Kumar, and L. A. Samuelson, Nano Lett. 2, 1273 (2002); H. Liu, J. Kameoka, D. A. Czaplewski, and H. G. Craighead, ibid. 4, 671 (2004).

${ }^{2}$ D. Li, Y. Wang, and Y. Xia, Nano Lett. 3, 1167 (2003); N. J. Pinto, A. T. Johnson, A. G. MacDiarmid, C. H. Mueller, N. Theofylaktos, D. C. Robinson, and F. A. Mirando, Appl. Phys. Lett. 83, 4244 (2003).

${ }^{3}$ M. M. Bergshoef and G. J. Vancso, Adv. Mater. (Weinheim, Ger.) 11, 1362 (1999).

${ }^{4}$ P. Gibson, H. Schreuder-Gibson, and D. Rivin, Colloids Surf., A 187-188, 469 (2001)

${ }^{5}$ D. Li and Y. Xia, Adv. Mater. (Weinheim, Ger.) 16, 1151 (2004), and references therein.

${ }^{6}$ M. M. Demir, M. A. Gulgun, Y. Z. Menceloglu, B. Erman, S. S. Abramchuk, E. E. Makhaeva, A. R. Khokhlov, V. G. Matveeva, and M. G. Sulman, Macromolecules 37, 573 (2004).

${ }^{7}$ S. W. Choi, S. M. Jo, W. S. Lee, and Y.-R. Kim, Adv. Mater. (Weinheim, Ger.) 15, 2027 (2003).

${ }^{8}$ D. A. Czaplewski, J. Kameoka, R. Maters, G. W. Coates, and H. G. Craighead, Appl. Phys. Lett. 83, 4836 (2003).

${ }^{9}$ H. Schmid and B. Michel, Macromolecules 33, 3042 (2000).

${ }^{10}$ A. Kumar and G. M. Whitesides, Appl. Phys. Lett. 63, 2002 (1993).

${ }^{11}$ E. Kim, Y. Xia, and G. M. Whitesides, Nature (London) 376, 581 (1995).

${ }^{12}$ K. Y. Suh, Y. S. Kim, and H. H. Lee, Adv. Mater. (Weinheim, Ger.) 13, 1386 (2001).

${ }^{13}$ Y. Xia and G. M. Whitesides, Angew. Chem., Int. Ed. Engl. 37, 550 (1998).

${ }^{14}$ D. Myers, Surface, Interface, and Colloids (Wiley, New York, 1999), pp. 97-118.

${ }^{15}$ Y. Xia, E. Kim, and G. M. Whitesides, Chem. Mater. 8, 1558 (1996).

${ }^{16}$ Y. Xia, J. J. McClelland, R. Gupta, D. Qin, X.-M. Zhao, L. Sohn, R. J. Celotta, and G. M. Whitesides, Adv. Mater. (Weinheim, Ger.) 9, 147 (1997); S. Y. Chou, P. R. Krauss, and P. J. Renstrom, Appl. Phys. Lett. 67, 3114 (1995).

${ }^{17}$ H. Hertz and J. Reine Angew. Math. 92, 156 (1882); K. R. Shull, Mater. Sci. Eng., R. 36, 1 (2002).

${ }^{18}$ S. Tan, R. L. Sherman Jr., and W. T. Ford, Langmuir 20, 7015 (2004).

${ }^{19}$ S. Madhugiri, A. Dalton, J. Gutierrez, J. P. Ferraris, and K. J. Balkus, J. Am. Chem. Soc. 125, 14531 (2003); W. R. Caseri, H. D. Chanzy, K. Feldman, M. Fontana, P. Smith, T. A. Tervoort, J. G. P. Goossens, E. W. Meijer, W. Egbert, A. P. H. J. Schenning, I. P. Dolbnya, M. G. Debije, M. P. de Hass, J. M. Warman, A. M. van de Craats, R. H. Friend, H. Sirringhaus, and N. Stutzmann, Adv. Mater. (Weinheim, Ger.) 15, 125 (2003).

${ }^{20}$ Z. Sun, E. Zussman, A. L. Yarin, J. H. Wendorff, and A. Greiner, Adv. Mater. (Weinheim, Ger.) 15, 1929 (2003); D. Li and Y. Xia, Nano Lett. 4, 933 (2004).

${ }^{21}$ R. H. Baughman, A. A. Zakhidov, and W. A. de Heer, Science 298, 787 (2002); J. Goldberger, R. He, Y. Zhang, S. Lee, H. Yan, H.-J. Choi, and P. Yang, Nature (Londion) / 422, 599 (2003). g/termsconditions. Downloaded to IP: 